\title{
Medication Errors: A Characterisation of Spontaneously Reported Cases in EudraVigilance
}

\author{
Victoria Newbould $^{1} \cdot$ Steven Le Meur ${ }^{2} \cdot$ Thomas Goedecke $^{1} \cdot$ Xavier Kurz $^{1}$
}

Published online: 11 July 2017

(c) The Author(s) 2017. This article is an open access publication

\begin{abstract}
Introduction Medication errors recently became the focus of regulatory guidance in pharmacovigilance to support reporting, evaluation and prevention of medication errors. Objective This study aims to characterise spontaneously reported cases of medication errors in EudraVigilance over the period 2002-2015 before the release of EU good practice guidance.

Methods Case reports were identified through the adverse reaction section where a Medical Dictionary for Regulatory Activities $\left(\right.$ MedDRA ${ }^{\circledR}$ ) term is reported and included in the Standardised MedDRA ${ }^{\circledR}$ Query (SMQ) for medication errors. These case reports were further categorised by MedDRA $^{\circledR}$ terms, geographical region, patient age group and Anatomical Therapeutic Chemical classification system of suspect medicinal product(s).

Results A total of 147,824 case reports were retrieved, 41,355 of which were from the European Economic Area (EEA). Approximately $60 \%$ of these case reports were retrieved with the narrow SMQ. The absolute number of medication error case reports and the proportion to the total number of reports in EudraVigilance increased during the
\end{abstract}

The original version of this article was revised due to a retrospective Open Access Order.

Victoria Newbould

victoria.newbould@ema.europa.eu

1 Inspections, Human Medicines Pharmacovigilance and Committees Division, Pharmacovigilance and Epidemiology Department, European Medicines Agency (EMA), London E14 5EU, UK

2 Information Management Division, Business Data and Analytics Department, European Medicines Agency (EMA), London E14 5EU, UK study period, with peaks seen around 2005 and 2012 for cases with EEA origin. Fifty-two percent of case reports in which age was provided occurred in adults, $30 \%$ in the elderly and $18 \%$ in children, with almost half of these in children aged 2 months to 2 years.

Conclusion Case reports of medication errors in EudraVigilance steadily increased between 2005 and 2015, the reasons for which may be multifactorial, including increased awareness, changes to the MedDRA ${ }^{\circledR}$ terminology and the 2012 EU pharmacovigilance legislation and associated guidance for stakeholders, or a generally increased risk for errors as more medications become available.

\section{Key Points}

EU pharmacovigilance legislation focuses on medication errors and increased error reporting to EudraVigilance

A new Standardised MedDRA ${ }^{\circledR}$ Query for medication errors facilitates data retrieval and analysis in this first comprehensive review

Further research to assess the impact of EU regulatory guidance on error prevention strategies is required

\section{Introduction}

The EU pharmacovigilance legislation [1] has put an increased emphasis on medication errors and the regulatory requirement for national drug regulatory authorities and the pharmaceutical industry to report adverse drug reactions 
(ADRs) caused by a medication error. Hence, medication errors are explicitly included in the ADR definition of Directive 2001/83/EC. In November 2015, the EU regulatory network published a Good Practice Guide (GPG) [2] to support the recording, coding, reporting and assessment of medication errors associated with suspected serious and non-serious adverse reactions to national pharmacovigilance databases and/or EudraVigilance, as applicable. EudraVigilance is the centralised European database for reporting and evaluating suspected adverse reactions of medicines and is maintained by the European Medicines Agency (EMA) on behalf of the EU regulatory network. The database contains electronic Individual Case Safety Reports (ICSRs), hereafter referred to as case reports from within and outside the $\mathrm{EEA}^{1}$, in line with the reporting rules defined in the guideline on good pharmacovigilance practices (GVP) Module VI [3]. Unlike medication errors associated with adverse reactions, errors with the potential for harm and those not resulting in adverse reactions (including intercepted errors) are recorded in the Periodic Safety Update Report (PSUR) and are not reportable to EudraVigilance as ICSRs.

The GPG aims to define and classify medication errors for pharmacovigilance purposes, to increase awareness of the reporting requirements among stakeholders, and to improve the quality of error reports in EudraVigilance. It includes supporting key pharmacovigilance activities ranging from detecting and evaluating signals of medication errors to implementation of risk minimisation and error prevention strategies [4]. The objective of this descriptive study was to investigate the frequency and patterns of case reports in EudraVigilance associated with medication errors during the period 2002-2015, before the release of the GPG. This is the first comprehensive quantitative review of medication errors in EudraVigilance and will thereby serve as a basis to further evaluate the implementation of the GPG recommendations and measure its impact on the reporting and prevention of medication errors. For the purpose of this study, we refer to the definition of a medication error provided in the GPG: " $a$ medication error is an unintended failure in the drug treatment process that leads to, or has the potential to lead to harm to the patient". The GPG further explains that a failure in the drug treatment process does not refer to lack

\footnotetext{
1 Pharmaceutical companies that hold the marketing authorisation of a medicine, as well as national medicines regulatory authorities, are legally required to submit reports of suspected side effects that occurred in the EEA to EudraVigilance, including reports received from healthcare professionals and patients. Pharmaceutical companies that hold the marketing authorisation for a medicine in the EEA are also legally required to submit to EudraVigilance all reports of suspected unexpected adverse reactions that are serious and that occurred in a third country (non-EEA) where they hold a marketing authorisation.
}

of efficacy of the drug but rather to human- or processmediated failures.

\section{Methods}

\subsection{Data Source}

For this study, spontaneous case reports in the EudraVigilance Post-Authorisation Module were used. The criteria for a valid case report and definitions of the ICSR data elements are specified in the International Conference on Harmonization (ICH) guidelines [5]. Valid case reports include, at a minimum, an identifiable reporter, an identifiable patient, and at least one drug and one ADR. The ICSR reaction section is coded with the Medical Dictionary for Regulatory Activities ${ }^{2}\left(\right.$ MedDRA $^{\circledR}$ ) terminology, but free text in the case summary narrative section may also provide a rich source of information on medication errors. MedDRA $^{\circledR}$ is organised into a hierarchy of five levels arranged by granularity, from very specific 'Low-Level Term' (LLT) to an entire 'System Organ Class' (SOC). EudraVigilance data can be retrieved and analysed using Standardised MedDRA ${ }^{\circledR}$ Queries (SMQs) developed by the Council for International Organisations of Medical Sciences (CIOMS), which combines terms related to a defined medical condition or area of interest that may be contained across different areas of the hierarchy [6, 7]. For our analysis, all case reports associated with a medication error were identified, where at least one MedDRA ${ }^{\circledR}$ LLT from the SMQ for medication errors (released in March 2016 with MedDRA $^{\circledR}$ version 19.0) has been coded in the adverse reaction section.

\subsection{Data Extraction}

The narrow SMQ for medication errors has 89 terms; additional terms are available to form the broad SMQ. Whereas the narrow terms are highly likely to represent the condition of interest (a 'narrow' scope), broad terms provide for retrieving additional potential cases of interest, although those cases may, on closer, inspection prove to be irrelevant for a particular condition. For example, there are several product-quality terms in the broad SMQ, which could possibly be associated with medication errors. Data were retrieved with both the narrow and broad SMQ for

\footnotetext{
2 MedDRA $^{\circledR}$, the Medical Dictionary for Regulatory Activities, is the international medical terminology developed under the auspices of the International Conference on Harmonisation of Technical Requirements for Registration of Pharmaceuticals for Human Use $(\mathrm{ICH})$. The MedDRA ${ }^{\circledR}$ trademark is owned by the International Federation of Pharmaceutical Manufacturers and Associations (IFPMA) on behalf of the ICH.
} 
medication errors. The possibility of retrieving duplicate cases was mitigated through semi-automated checks for duplicate case reports performed by the EMA according to a defined algorithm, and managed through a process of merging duplicate cases [8]. Data were retrieved for cases reported between 1 January 2002 and 31 December 2015 using EudraVigilance 'ICSR message type' based on the gateway date, and for serious and non-serious reports. Nonserious PSUR reports submitted in the past on a voluntary basis were excluded. Case reports were filtered by narrow or broad SMQ and by region of occurrence, i.e. European Economic Area (EEA) versus non-EEA origin, and by United Nations geo-scheme, which divides the countries of the world into regional and subregional groups [9]. The Anatomical Therapeutic Chemical (ATC) Classification System code of the suspect medicinal products in the reports was also retrieved. The full ICSR data extraction pathway is shown in Fig. 1.

\section{Results}

A total of 147,824 case reports were retrieved, of which 41,355 refer to cases occurring within the EEA. Approximately $60 \%$ of these are narrow SMQ cases. A case report may contain more than one MedDRA ${ }^{\circledR}$ term related to a medication error. The absolute number of medication error case reports has been increasing over time between 2002 and 2015, as shown in Fig. 2. The proportion of medication errors to total number of ADR reports has also been increasing, with peaks seen around 2005 and 2012 for cases with EEA origin (Fig. 3). There have also been increases over time in the proportion of medication error reports compared with the total number for non-EEA cases, although the proportion is lower, and no peak was seen in 2012. Using the narrow SMQ and looking at absolute numbers, $59 \%$ of medication error cases in EudraVigilance are from North and South America. For Europe, 60\% are from Western Europe, 24\% from Northern Europe, 12\% from Southern Europe, and 4\% from Eastern Europe. In the EEA, the most frequently reported MedDRA ${ }^{\circledR}$ Preferred Term (PT) is 'Medication error', followed by 'Accidental overdose', 'Inappropriate schedule of drug administration' and 'Incorrect dose administered' (Fig. 5). Eight percent of cases are from Asia, and only 2 and $1 \%$ are from Oceania and Africa, respectively (Fig. 6). Despite the low numbers from Oceania and Africa, the cumulative total proportions of medication errors versus all reported cases are higher than in Europe. The proportion of errors of all reported ADRs is approximately $3 \%$ for Eastern, Western and Southern Africa, although this figure is lower for Northern Africa (1.5\%) and much lower for Middle Africa $(0.7 \%)$. The patterns of errors in Africa vary with respect to the general global pattern, and also across the African regions.

As shown in Table 1, the ranking for ATC codes associated with medication errors reported inside and outside the EEA is largely similar, with vaccines ranking higher in the EEA (vaccines rank at number 12 outside the EEA). The most commonly reported PT for vaccines is 'Inappropriate schedule of drug administration'. Drilling down into further detail of the most reported errors inside and outside the EEA combined, accidental overdoses occurred most frequently with paracetamol, opiates and benzodiazepines, while drug administration errors were most frequently reported with cisapride, insulin, fluticasone/salmeterol, fentanyl and salbutamol.
Fig. 1 EudraVigilance ICSR data extraction pathway using the broad and narrow SMQ for medication errors. Non-serious PSUR cases were excluded. ICSR individual case safety reports, $S M Q$ standardised MedDRA $^{\circledR}$ Query, EVPM eudravigilance postauthorisation module, $P T$ preferred terms, EEA European economic area, $A T C$ anatomical therapeutic classification, PSUR periodic safety update report, UN United Nations, MedDRA ${ }^{\circledR}$ medical dictionary for regulatory activities

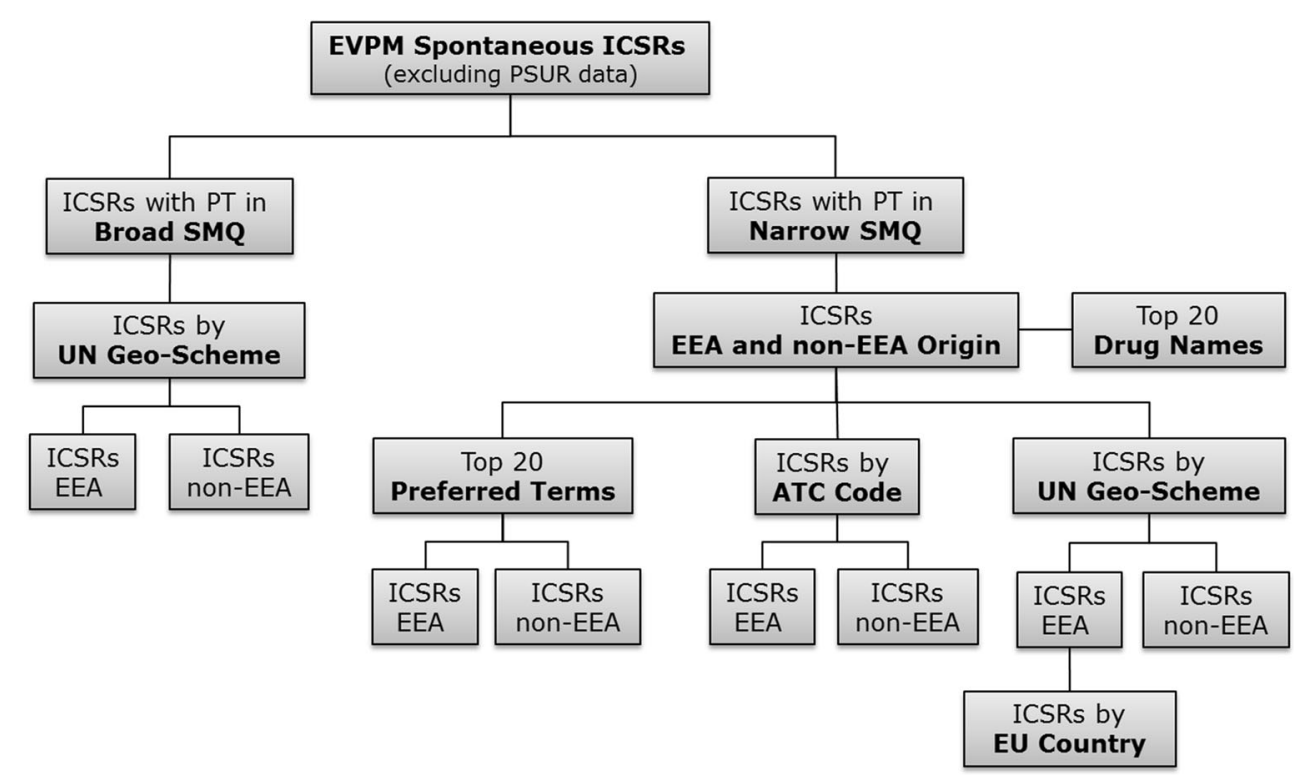


Fig. 2 Number of ICSRs for medication errors reported in the EEA and globally (includes both EEA and non-EEA cases) to EudraVigilance between 2002 and 2015. ICSRs individual case safety reports, $S M Q$ Standardised MedDRA ${ }^{\circledR}$ Query, EEA European economic dictionary for regulatory activities retrieved with the narrow SMQ area, MedDRA ${ }^{\circledR}$ medical

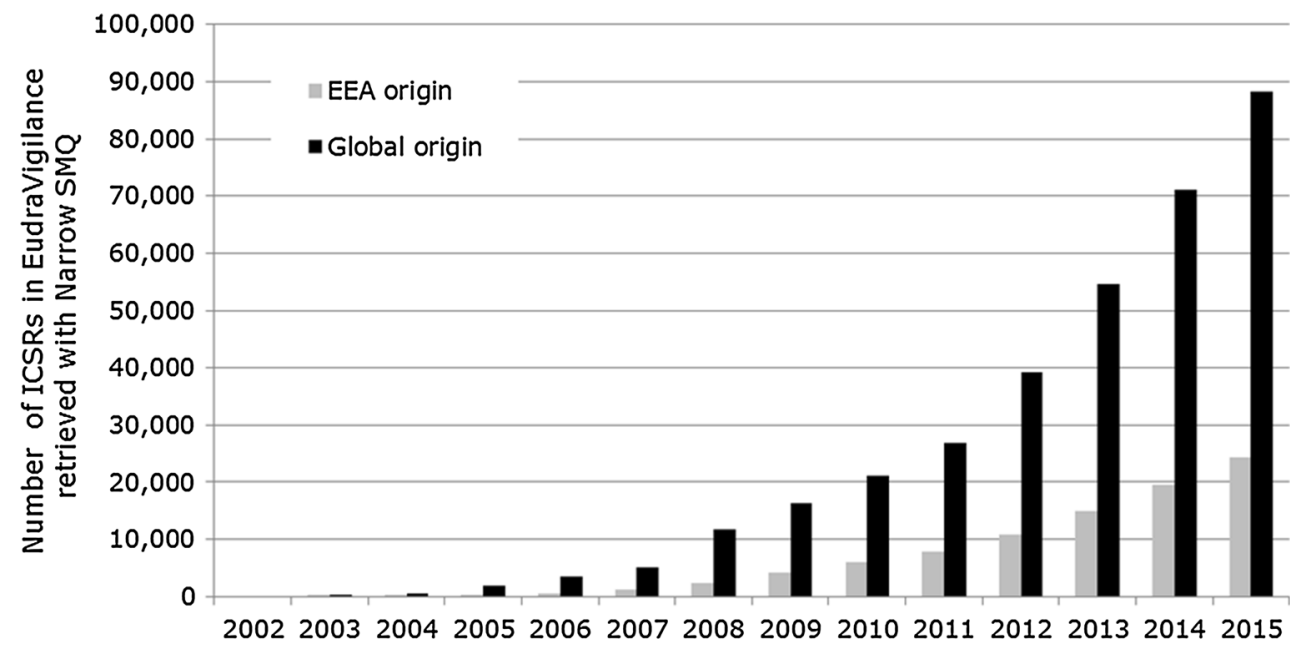

Fig. 3 Proportion of medication error ICSRs with EEA origin retrieved with the broad and narrow SMQ to the total number of ICSRs reported to EudraVigilance between 2002 and 2015. The broad SMQ includes the terms from the narrow SMQ. ICSRs Individual Case Safety Reports, EEA European Economic Area, $S M Q$ Standardised MedDRA ${ }^{\circledR}$ Query, $\operatorname{MedDR} A^{\circledR}$ Medical Dictionary for Regulatory Activities

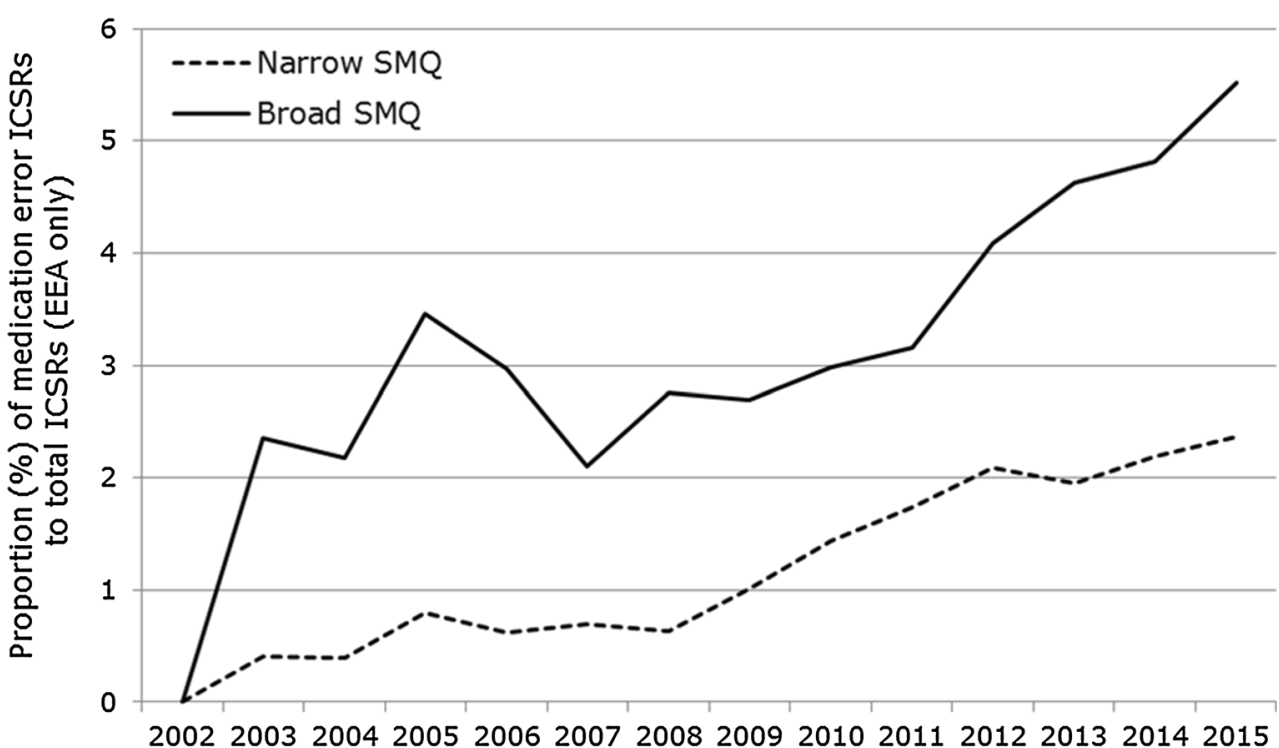

Table 1 Ranking of ATC codes most frequently reported with medication errors by EEA and non-EEA origin

\begin{tabular}{lll}
\hline Ranking & EEA & Non-EEA \\
\hline 1 & J07 (Vaccines) & N02 (Analgesics) \\
2 & N05 (Psycholeptics) & N05 (Psycholeptics) \\
3 & N02 (Analgesics) & A10 (Drugs used in diabetes) \\
4 & B01 (Antithrombotics) & B01 (Antithrombotics) \\
5 & G03 (Sex hormones) & L04 (Immunosupressants) \\
6 & N06 (Psychoanaleptics) & R03 (Obstructive airways disease) \\
7 & A10 (Drugs used in diabetes) & N06 (Psychoanaleptics) \\
8 & N03 (Antiepileptics) & G03 (Sex hormones) \\
9 & L01 (Antineoplastics) & N03 (Antiepileptics) \\
10 & J01 (Antibacterials for systemic use) & N07 (Other nervous system drugs) \\
\hline
\end{tabular}

ATC anatomical therapeutic chemical, EEA European economic area
Incorrect dose administered was highly associated with rivaroxaban, insulin, mifepristone, misoprostol and paracetamol .
In the EEA, for case reports where an age was provided, $30 \%$ of errors occurred in the elderly (over 64 years of age), 52\% were in the age category $18-64$ years, and $18 \%$ 
occurred in children ( $<17$ years of age), with almost half of these in the age category 2 months to 2 years.

\section{Discussion}

Our study demonstrates that reporting of cases of medication errors has been steadily increasing between 2005 and 2015, both in absolute numbers and proportion to all other case reports in EudraVigilance. The observed reporting trends may be explained by multiple factors. First, there have been significant transformations to the MedDRA $^{\circledR}$ hierarchy since 2005 , expanding from a single term for medication error, to a fully conceptual approach, to coding a broad range of medication errors, including coding examples and a clear distinction from product quality issues. Relevant guidance for MedDRA ${ }^{\circledR}$ coders [10] was updated accordingly, which may have contributed to more accurate coding of ADR reports associated with medication errors, where previously the error may have just been mentioned in the free-text narrative. Secondly, stakeholder awareness of the need to code medication errors in ADR reports for pharmacovigilance purposes has increased, which appears to be substantiated by the 2005 peak in reporting of both narrow and broad SMQ terms (Fig. 3). However, the 2005 peak is not seen for non-EEA cases (Fig. 4), and it is also known that EEA reporting trends generally peaked in 2005 after the EU enlargement and after mandatory electronic ADR reporting to EudraVigilance coming into effect. Another reporting peak in 2012 (Fig. 4), for EEA cases only, may be explained by the ADR definition of the 2012 pharmacovigilance legislation explicitly including medication errors. Alternatively, actual error rates may have increased as new medicinal products in the same indications, but different strengths or formulations compared with established treatment options, became available (e.g. novel high-strength insulin products or fixed-combination insulin products), or due to more medicines with complex methods of use (e.g. requiring drug delivery devices).

The results of our study serve as a baseline to further evaluate the impact of the changes in regulatory requirements for medication errors and the GPG recommendations, including a reference for future trends with a potential to prevent errors for the benefit of public health, as shown by the ATC codes with the highest medication error rates reported in Table 1 .

The reporting incidence of specific MedDRA ${ }^{\circledR}$ PTs requires careful interpretation. As shown in Fig. 5, the most frequently reported PT is 'Medication error', and, considering the gradual expansion of the terminology described earlier, this observation may be attributable to cases reported at the time when only this single term was available. Changing reporting practices and refined coding concepts also need to be taken into account. For example, in the EEA, the frequently reported PT 'Inappropriate schedule of drug administration' refers predominantly to non-reportable case reports formally sent as expedited spontaneous cases to EudraVigilance instead of 'PSURtype' cases. Despite medication errors without adverse reaction(s) not being reportable to EudraVigilance, approximately $7 \%$ of the retrieved case reports have no adverse reaction included, although we did not perform an assessment of the report quality. The quality of coding is monitored as part of the data quality checking procedures referred to in the guideline on GVP Module VI [3].
Fig. 4 Proportion of medication error ICSRs retrieved with the narrow SMQ to the total number of ICSRs reported to EudraVigilance between 2002 and 2015 with EEA and non-EEA origin. ICSRs individual case safety reports, $S M Q$ Standardised MedDRA $^{\circledR}$ Query, EEA European Economic Area, $M e d D R A^{\circledR}$ Medical Dictionary for Regulatory Activities

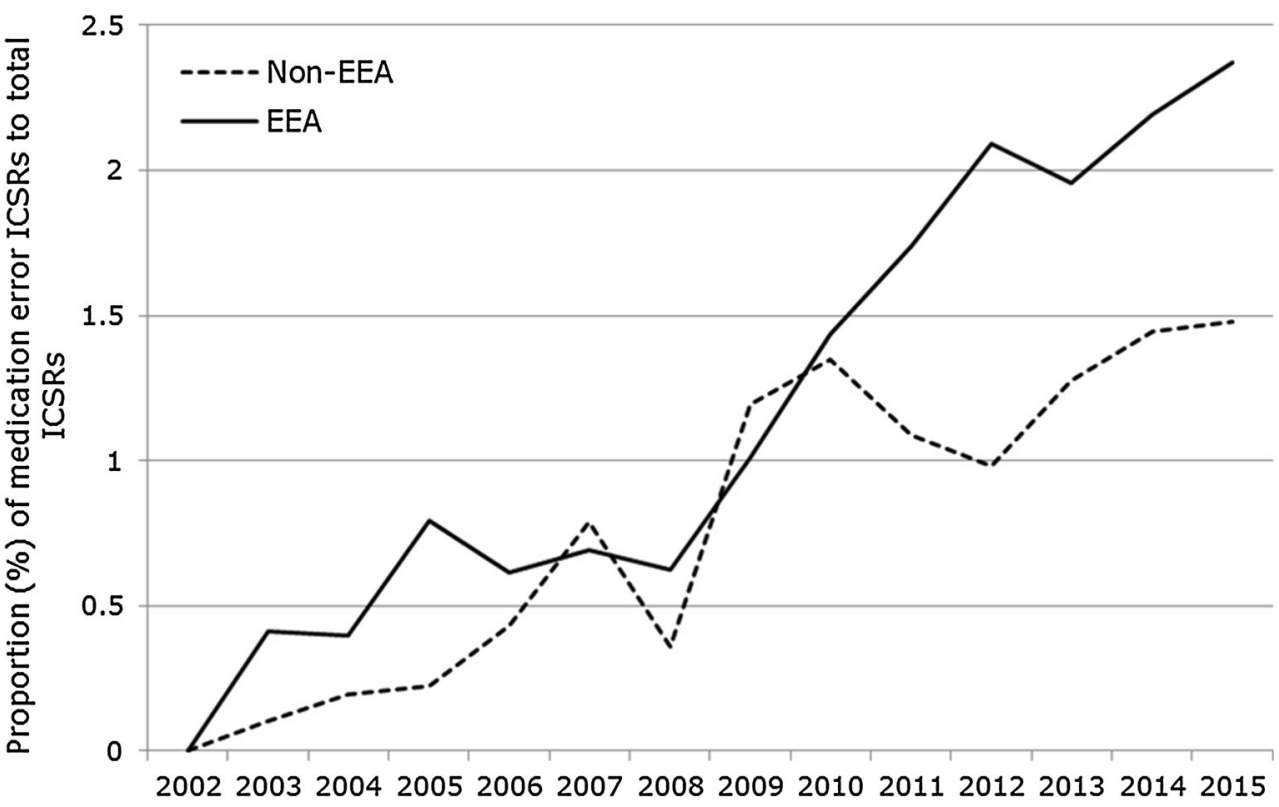




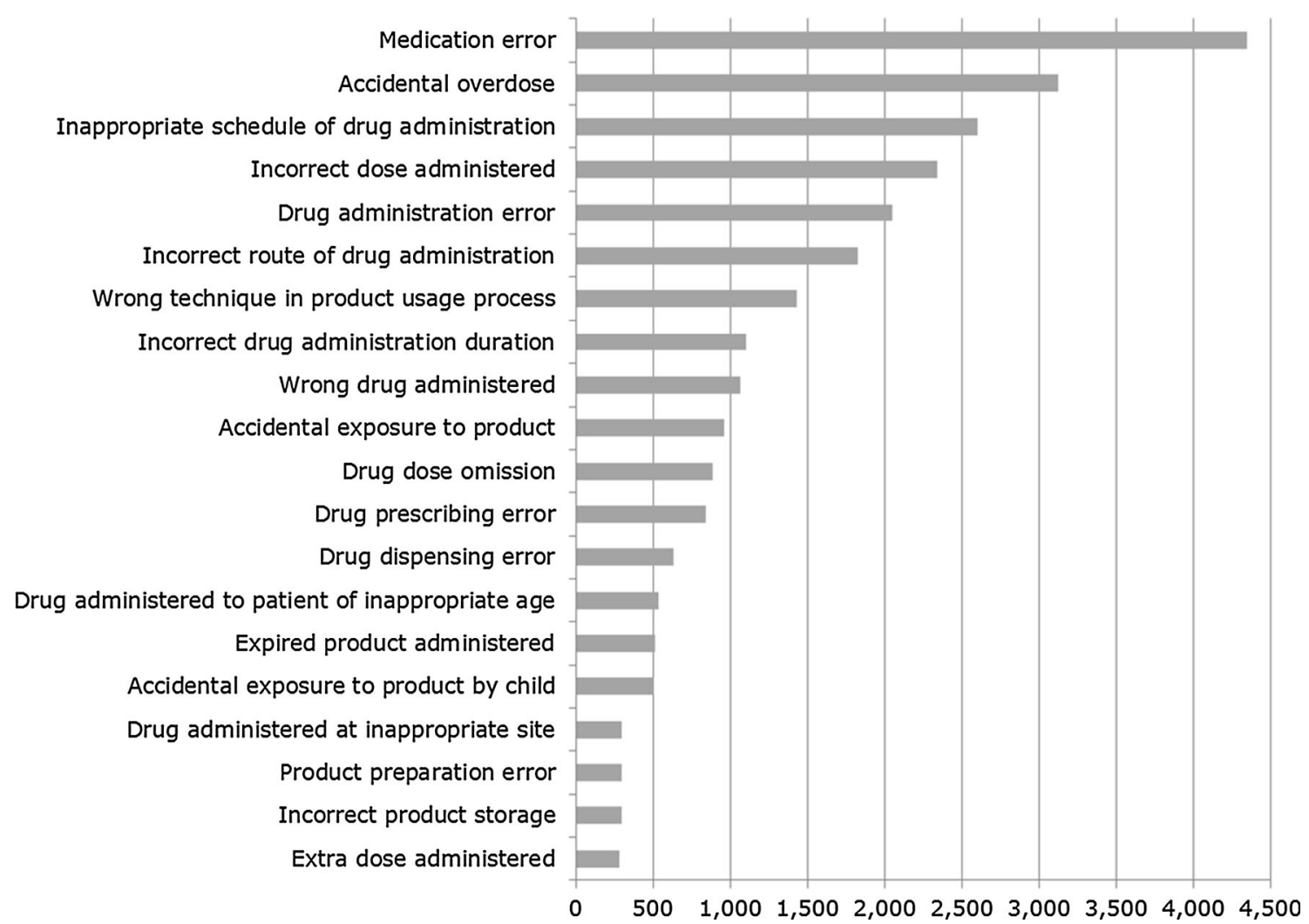

Fig. 5 Top 20 reported MedDRA ${ }^{\circledR}$ Preferred Terms from the narrow Standardised MedDRA ${ }^{\circledR}$ Query in Individual Case Safety Reports with European Economic Area origin $(n=25,865)$. $M e d D R A^{\circledR}$ Medical Dictionary for Regulatory Activities

The distribution of medication error reports across geographical regions is shown in Fig. 6. The interpretation needs to take into account different regional pharmacovigilance practices and reporting requirements for medication errors that are likely to follow general ADR reporting trends in these ICH regions. Although the focus of this study is on European data, the African cases are noteworthy given a fairly high proportion of $2.23 \%$ of all reported cases are medication errors, but overall reporting is significantly lower compared with other regions, and different types of errors predominate compared with global patterns. Preparation and storage errors are most frequent in Middle Africa, whereas in Eastern Africa labelled interactions (monitoring errors) dominate. A survey in Nigeria [11] concluded that the prevalence of medication errors is high among healthcare professionals due to knowledge gaps and practice deficiencies that require interventions.

The breakdown in Western, Eastern, Northern and Southern Europe follows reporting trends in EudraVigilance as a whole, where ADR reporting is generally higher in Western Europe given the higher population numbers and longer duration of EU/EEA membership. However, adjustment for population size still indicates a wide variance in reporting rates across EU Member States during the most recent reporting period.

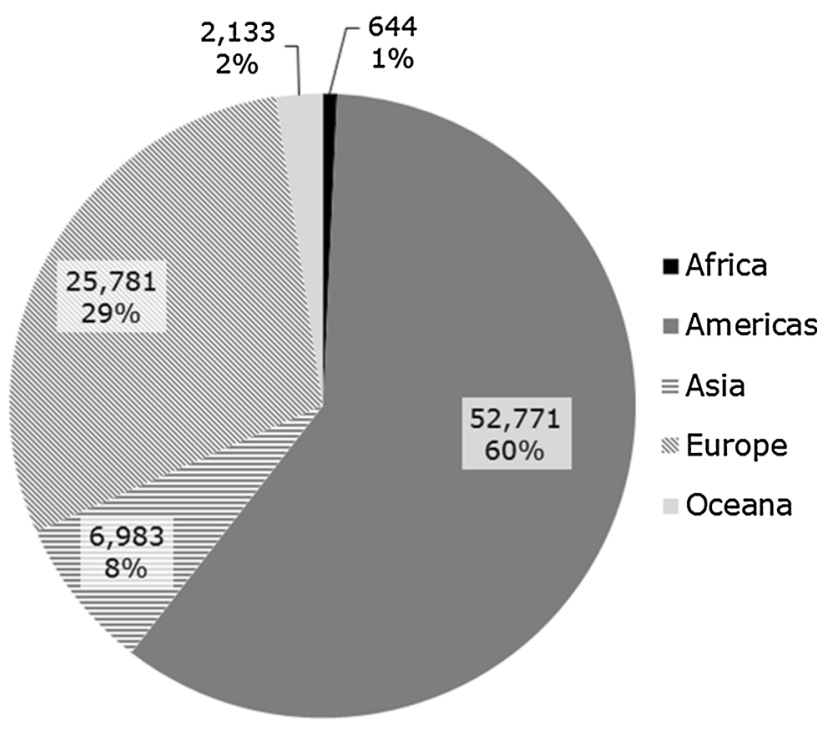

Fig. 6 Distribution of medication error Individual Case Safety Reports with narrow Standardised MedDRA ${ }^{\circledR}$ Query by geographic region $(n=88,318)$, at 31 December 2015. MedDRA ${ }^{\circledR}$ Medical Dictionary for Regulatory Activities

An analysis of age categories associated with medication errors is expected to show higher rates in the elderly or in children compared with the general population, but 
differences from general ADR reporting trends in these populations warrant further research, including, for particular products, increasing the risk of errors, e.g. vaccines for infants or commonly co-prescribed medicines in the elderly.

Table 1 shows the ranking of medication errors by ATC code. For therapeutic classes such as analgesics, psycholeptics and antidiabetics, ranking among the top 10 most error-prone medicines would be expected, but not for other therapeutic classes. The fact that medication errors with vaccines rank top for EEA data should be interpreted with caution. The reporting patterns for vaccines are largely similar for EEA and non-EEA cases, with 'Inappropriate schedule of drug administration' most frequently reported, followed by 'Incorrect route of drug administration' and 'Drug administered to patient of inappropriate age'. It appears that 'Expired product administered' and 'Incomplete course of vaccination' are reported more frequently in the EEA, although further analysis is required as this classification is based on the ATC code, where it may not always have been provided in the medicinal product dictionary. Any differences in EEA and non-EEA reporting of vaccine cases may have various explanations, e.g. vaccination uptake and reporting programmes are different across the Member States in the EEA, and different compared with the rest of the world. It is challenging to compare data from the EEA with non-EEA as the drug utilisation patterns vary. In addition, miscoding and incorrectly reported cases need to be taken into account.

The high numbers of case reports of 'Drug administration error' with cisapride are noteworthy in the context of signal detection and assessment of medication error reports. Measures of disproportionality of reporting to detect safety signals in EudraVigilance are not applicable to medication errors and alternative methods such as root cause analysis are warranted to assess the causes and circumstances in clinical practice. Most of the administration errors reported for cisapride were litigation cases in relation to QT prolongation, where, in addition to QT prolongation, a term referring to medication errors had been coded, implying liability aspects. The potential for litigation cases to skew data sets for signal detection purposes is recognised and proposals were made to exclude these cases from analyses [12] which may be particularly relevant for medication errors.

Another example is the high number of case reports with 'Incorrect dose administered' for rivaroxaban, which is marketed in different dosing schedules for different indications, including loading and maintenance doses for one indication and the need for dose adjustment in patients with renal impairment. In some case reports the dose had been adjusted when it should not have been, or vice versa, and in some cases patients had taken the wrong dose. We also found mixed approaches to coding, with some cases misclassified as off-label use or misuse, and this product is a good example for a potential error due to complicated dosing schedules. To adequately manage the risk of medication errors with rivaroxaban, a prescriber guide and patient alert card have been introduced to ensure correct administration, and these measures were also subject to effectiveness evaluation.

We did not assess whether individual case reports were truly medication errors or potentially miscoded, and no analysis of the root causes or associated harms could be performed given the large amount of data. In the context of routine EU pharmacovigilance activities for medication errors included in this review, regulatory actions may have been taken as appropriate, either at a national or EU level. Pharmacovigilance Risk Assessment Committee (PRAC) recommendations for any additional measures to prevent medication errors for centrally authorised medicines are regularly published on the EMA website to raise awareness among patients, carers and healthcare professionals [13]; however, the long-term impact of the GPG on pharmacovigilance, regulatory decision making and error-prevention strategies remains to be further investigated.

\section{Conclusions}

Reporting of medication errors has increased over the analysis period 2002 to end 2015. The release of the MedDRA $^{\circledR}$ SMQ for medication errors has been an important milestone to improve the detection and retrieval of medication errors in EudraVigilance. In Europe, the number of reports have steadily increased since 2008, with a peak around 2012. Several factors could be responsible for this increase: expanded MedDRA ${ }^{\circledR}$ terminology and guidance; increased awareness of the need to report medication errors for pharmacovigilance purposes; and the public consultation and release of EU guidance on coding, recoding, reporting and assessment of medication errors, and the guidance on risk minimisation and prevention of medication errors. In addition, in 2015 the EMA launched a dedicated webpage to communicate to patients and healthcare professionals, as well as the general public, important risk minimisation activities in relation to medication errors for centrally authorised medicinal products. The synergy of these initiatives at EU regulatory level, and globally at ICH level, seem likely to have contributed to this increase and also to the granularity of coding of medication error reports associated with adverse reactions to EudraVigilance. Besides these likely effects, there is a possibility that actual error rates have increased as more generic medicines are launched, and novel medicines with increasingly complex instructions for use. This study is 
intended to provide a baseline for the reporting of medication errors to EudraVigilance. The impact of the GPG on the pharmacovigilance of medication errors and data quality to allow for complementary analysis (e.g. of the root causes) remain to be further explored.

\section{Compliance with Ethical Standards}

Funding No sources of funding were used to assist in the preparation of this article.

Conflicts of interest Victoria Newbould, Steven Le Meur, Thomas Goedecke and Xavier Kurz have no conflicts of interest that are directly relevant to the content of this study.

Disclaimer The views expressed in this article are the personal views of the authors and may not be understood or quoted as being made on behalf of or reflecting the position of the EMA or one of its committees or working parties.

Open Access This article is distributed under the terms of the Creative Commons Attribution-NonCommercial 4.0 International License (http://creativecommons.org/licenses/by-nc/4.0/), which permits any noncommercial use, duplication, adaptation, distribution and reproduction in any medium or format, as long as you give appropriate credit to the original author(s) and the source, a link is provided to the Creative Commons license and any changes made are indicated.

\section{References}

1. Union European. Directive 2001/83/EC of the European Parliament and of the Council of 6 November 2001 on the Community code relating to medicinal products for human use. OJ. 2001;44(L311):67-128.

2. Pharmacovigilance Risk Assessment Committee. Good practice guide on recording, coding, reporting and assessment of medication errors (EMA/762563/2014). http://www.ema.europa.eu/ docs/en_GB/document_library/Regulatory_and_procedural_guide line/2015/11/WC500196979.pdf (2015). Accessed 15 Jun 2017.

3. European Medicines Agency. Guideline on good pharmacovigilance practices (GVP) Module VI-management and reporting of adverse reactions to medicinal products (Rev 1) (EMA/873138/ 2011 Rev 1). http://www.ema.europa.eu/docs/en_GB/document_
library/Scientific_guideline/2014/09/WC500172402.pdf (2014). Accessed 15 Jun 2017.

4. Goedecke T, Ord K, Newbould V, Brosch S, Arlett P. Medication errors: new EU good practice guide on risk minimisation and error prevention. Drug Saf. 2016;39(6):491-500.

5. E2B(R3) Individual Case Safety Report (ICSR). Specification and related files. http://estri.ich.org/e2br3/index.htm. Accessed 19 Jun 2017.

6. International Council for Harmonisation of Technical Requirements for Registration of Pharmaceuticals for Human Use (ICH). Medical dictionary for regulatory activities $\left(\right.$ MedDRA $\left.{ }^{\circledR}\right)$. Standardised MedDRA ${ }^{\circledR} \quad$ Queries. http://www.meddra.org/ standardised-meddra-queries (2016). Accessed 15 Jun 2017.

7. Development and rational use of Standardised MedDRA Queries (SMQs): retrieving adverse drug reactions with MedDRA. 2nd ed. Geneva: Council for International Organisations of Medical Sciences (CIOMS); 2016.

8. Committee for Medicinal Products for Human Use. CHMP guideline on detection and management of duplicate individual cases and Individual Case Safety Reports (ICSRs) (EMA/13432/ 2009). http://www.ema.europa.eu/docs/en_GB/document_library/ Regulatory_and_procedural_guideline/2012/06/WC500129037.pdf (2009). Accessed 15 Jun 2017.

9. United Nations Statistics Division. Composition of macro geographical (continental) regions, geographical sub-regions, and selected economic and other groupings. http://unstats.un.org/ unsd/methods/m49/m49regin.htm (2017). Accessed 15 Jun 2017.

10. International Council for Harmonisation of Technical Requirements for Registration of Pharmaceuticals for Human Use (ICH). MedDRA $^{\circledR}$ Term selection: points to consider. ICH-endorsed guide for MedDRA ${ }^{\circledR}$ Users. Release 4.12 based on MedDRA ${ }^{\circledR}$ Version 19.1. http://www.meddra.org/sites/default/files/guidance/ file/9491-1910_termselptc_r4_12_sep2016.pdf (2016). Accessed 15 Jun 2017.

11. Ogunleye O, Oreagba IA, Falade C, Isah A, Enwere O, Olayemi $\mathrm{S}$, et al. Medication errors among health professionals in Nigeria: a national survey. Int J Risk Saf Med. 2016;28(2):77-91.

12. European Medicines Agency. Screening for adverse reactions in EudraVigilance (EMA/849944/2016). http://www.ema.europa. eu/docs/en_GB/document_library/Other/2016/12/WC500218606. pdf (2016). Accessed 15 Jun 2017.

13. European Medicines Agency. Recommendations on medication errors. http://www.ema.europa.eu/ema/index.jsp?curl=pages/ regulation/document_listing/document_listing_000398.jsp\&mid= WC0b01ac058098f1c0 (2016). Accessed 15 Jun 2017. 